\title{
OPTICAL IDENTIFICATIONS OF COMPACT RADIO SOURCES
}

\author{
G. G. POOLEY
}

Cavendish Laboratory, Cambridge, Great Britain

\begin{abstract}
A study of twelve compact radio sources whose radio spectra show pronounced peaks at centimetre wavelengths has been made using the Cambridge onemile radio telescope operating at a frequency of $5 \mathrm{GHz}$. For each of these sources we have measured accurate positions to closer than one arc sec and determined upper limits to their angular sizes.

A search for optical identifications using the prints of the Sky Survey has revealed probable candidates for eleven of the twelve sources, an unusually high success rate. Of the suggested identifications, three are galaxies (OC $328=4 \mathrm{C} 31.04$, VRO 20.04.02, OQ 208), and eight are possible or confirmed QSOs (NRAO $140=4 \mathrm{C} 32.14=$ OE 355 , OI 315, P 0735 + 17 = VRO 17.07.02, OI 363, OJ 287=VRO 20.08.01, OK 290, OR 103, DA 406). Details are given by G. M. Blake, Astrophys. Letters 6, 201 (1970).
\end{abstract}

